\title{
Safety and effectiveness evaluation of a two-handed technique combining harmonic scalpel and laparoscopic Peng's multifunction operative dissector in laparoscopic hemihepatectomy
}

\author{
Jingwei Cai ${ }^{\dagger}$, Guixing Jiang ${ }^{\dagger}$, Yuelong Liang, Yangyang Xie, Junhao Zheng and Xiao Liang*
}

\begin{abstract}
Objectives: This study was designed to evaluate the safety and effectiveness of a two-hand technique combining harmonic scalpel (HS) and laparoscopic Peng's multifunction operative dissector (LPMOD) in patients who underwent laparoscopic hemihepatectomy $(\mathrm{LHH})$.

Methods: We designed and conducted a case-control study nested in a prospectively collected laparoscopic liver surgery database. Patients who underwent LHH for liver parenchyma transection using HS + LPMOD were defined as cases $(n=98)$ and LPMOD only as controls $(n=47)$ from January 2016 to May 2018. Propensity score matching (1:1) between the case and control groups was used in the analyses.

Results: The case group had significantly less intraoperative blood loss in milliliters (169.4 \pm 133.5 vs. $221.5 \pm 176.3$, $P=0.03)$ and shorter operative time in minutes $(210.5 \pm 56.1$ vs. $265.7 \pm 67.1, P=0.02)$ comparing to the control group. The conversion to laparotomy, postoperative hospital stay, resection margin, the mean peak level of postoperative liver function parameters, bile leakage rate, and others were comparable between the two groups. There was no perioperative mortality.

Conclusions: We demonstrated that the two-handed technique combing HS and LPMOD in LHH is safe and effective which is associated with shorter operative time and less intraoperative blood loss compared with LPMOD alone. The technique facilitates laparoscopic liver resection and is recommended for use.
\end{abstract}

Keywords: Laparoscopic hemihepatectomy, Harmonic scalpel, Laparoscopic Peng's multifunction operation dissector, Two-handed technique

\footnotetext{
*Correspondence: srrshlx@zju.edu.cn

${ }^{\dagger}$ Jingwei Cai and Guixing Jiang contributed equally to this work.

Department of Hepatobiliary and Pancreatic Surgery, Sir Run Run Shaw Hospital, Zhejiang University School of Medicine, 3 East Qingchun Road,

Hangzhou 310016, People's Republic of China
}

(c) The Author(s). 2021 Open Access This article is licensed under a Creative Commons Attribution 4.0 International License, which permits use, sharing, adaptation, distribution and reproduction in any medium or format, as long as you give appropriate credit to the original author(s) and the source, provide a link to the Creative Commons licence, and indicate if changes were made. The images or other third party material in this article are included in the article's Creative Commons licence, unless indicated otherwise in a credit line to the material. If material is not included in the article's Creative Commons licence and your intended use is not permitted by statutory regulation or exceeds the permitted use, you will need to obtain permission directly from the copyright holder. To view a copy of this licence, visit http://creativecommons.org/licenses/by/4.0/ The Creative Commons Public Domain Dedication waiver (http://creativecommons.org/publicdomain/zero/1.0/) applies to the data made available in this article, unless otherwise stated in a credit line to the data. 


\section{Introduction}

Massive bleeding is a big challenge for hepatic resection, especially hemihepatectomy. It is a cause of death during the surgery and hemorrhage after the surgery and affects prognosis as well. Increased intraoperative bleeding during liver surgery has been reported to have a negative impact on postoperative recovery and prognosis [1]. Massive blood loss during liver surgery is related to the high risk of postoperative mortality and recurrence of hepatocellular carcinoma. Massive intraoperative blood loss may lead to a longer time of systemic hypoperfusion and affects oxygen delivery to vital organs [2]. In addition, perioperative blood transfusion due to massive blood loss is associated with worse survival outcomes in postoperative patients [3, 4]. Therefore, techniques which can help minimize bleeding during the hepatic resection are demanded to be developed.

Over the last two decades, laparoscopic surgery has been employed in various surgical fields [5-9]. With the development of laparoscopic techniques, laparoscopic liver resection has widely been carried out with the feasibility, safety, oncological efficiency, and surgical indications [10, 11]. Laparoscopic hemihepatectomy (LHH) was associated with less intraoperative blood loss, better postoperative recovery, and a shorter length of hospital stay. Safe and effective parenchyma transection is a critical step in LHH, which is dependent on the efficient management of the parenchyma division and hemostasis. Previous studies have compared the clinical benefits of different methods of hepatic transection in open hepatectomy $[12,13]$. No standardized or best method, however, has been proposed for LHH to date and the appropriate laparoscopic surgical instruments for transecting the liver parenchyma are still controversial. The objective of this study was to report a novel simple method to perform laparoscopic hemihepatectomy and to explore the feasibility of this new technique.

\section{Methods}

\section{Study design and patients}

A retrospective case-control study nested in a cohort study was conducted using prospectively collected data from medical records system in the Sir Run Run Shaw Hospital at Zhejiang University School of Medicine from January 2016 to May 2018. All data were entered into the database by Dr. JHZ. All patients were given and signed the consent form. Patients who underwent LHH during the study period by two-handed liver surgery technique HS + LPMOD were defined as cases and LPMOD only as controls. Patients who received the liver wedge resection, liver segment resection, and extended hemihepatectomy were excluded from this study. In total, 497 patients were excluded, and 145 patients were included in the study. To be more specific, 497 cases were excluded due to unclear hepatectomy type $(n=32)$, missing hepatectomy type $(n=10)$, partial hepatectomy $(n$ $=330)$, and 125 segmental hepatectomy $(n=125)$. Of the 145 patients included in the study, 98 patients underwent the liver parenchyma transection HS plus LPMOD method while 47 underwent LPMOD only.

\section{Surgical procedures}

Several critical surgical instruments used in LHH were shown in Fig. 1. Patients should be placed in the supine position. Three surgeons including one primary surgeon and two assistants were needed. The primary surgeon

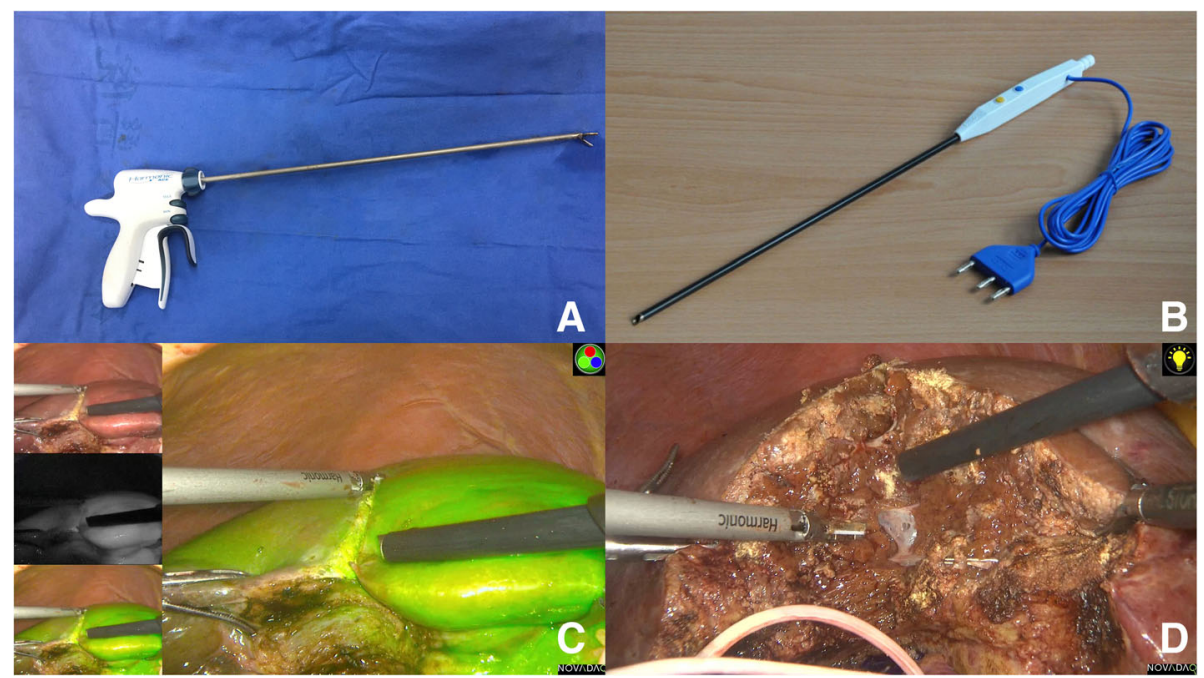

Fig. 1 Some critical surgical instruments used in laparoscopic hemihepatectomy (LHH). A Harmonic scalpel (HS). B Laparoscopic Peng's multifunction operative dissector (LPMOD). C Liver parenchyma transection was started with HS. D Liver parenchyma is crushed and divided by LPMOD combined with HS and intrahepatic ducts and vessels were observed 
stood on the left side of the patient. Carbon dioxide pneumoperitoneum was set at $10-14 \mathrm{mmHg}$. LHH was routinely performed with a four-port method. The observation port was placed above the umbilicus $(10 \mathrm{~mm})$; the main operating port was put below the xiphoid process $(12 \mathrm{~mm})$ and on the right collarbone midline $(12 \mathrm{~mm})$; and the assistant port was put on the right axillary frontline $(5 \mathrm{~mm})$.

Following routine laparoscopic exploration, intraoperative sonography was used to confirm the extent of diseases and the relationship of important vessels and guide the appropriate parenchyma transection plane. Hepatoduodenal ligament occlusion was performed routinely by the Pringle maneuver to occlude the hepatic inflow for $10 \mathrm{~min}$ and released for $5 \mathrm{~min}$ [14].

In the HS + LPMOD group, the primary surgeon needed to operate with both hands: the left hand held the HS, and the right hand held LPMOD to dissect the parenchyma. The HS was applied to crack liver capsule approximately $2 \mathrm{~cm}$ away from the superficial liver tissues, while the LPMOD was simultaneously used to expose, electrically coagulate, and suction. Alternated operation of two hands was performed to cut and crush the liver parenchyma in different parts of the transection plane. Vessels less than $3 \mathrm{~mm}$ were directly sealed by HS. Liver parenchyma hemorrhage was managed by LPMOD.

In the LPMOD only group, the technique for LHH was described previously in our institution [15]. Briefly, liver parenchyma is crushed and aspirated, and then intrahepatic ducts and vessels can be dissected and preserved safely for clipping or ligation. Vessels less than 3 $\mathrm{mm}$ would be electrically coagulated by LPMOD.

In both groups, the vessels larger than $3 \mathrm{~mm}$ were cut by HS after clipping by Hem-lock or titanium clap on the remnant side. Laparoscopic vascular stapler was adopted to divide hepatic pedicles or some hepatic veins which were larger than $10 \mathrm{~mm}$.

Evaluated endpoints were operative outcomes including the type of liver resection, intraoperative blood loss, blood transfusion, operative time, conversion to laparotomy, postoperative hospital days, hospital mortality, resection margin, postoperative bile leakage, and postoperative liver function. The evaluation of postoperative liver function was assessed by measurements of postoperative mean peak level of alanine aminotransferase (ALT), aspartate transaminase (AST), total bilirubin (TBIL), and prothrombin time (PT). Biliary fistula was defined as bilious drainage lasting more than 7 days after the surgery [16].

\section{Statistical analysis}

All analyses were performed using the SPSS 22.0 statistical software. Continuous variables were expressed by mean and standard deviation (SD), and categorical variables were expressed by number and percentage for each category. Paired-sample $t$ test or Wilcoxon ranksum test was used for continuous variables. And Pearson chi-square test or Fisher's exact test was used for categorical data. To eliminate some potential biases due to confounding factors, the propensity score matching (PSM) technique was used. A matching ratio of 1 to 1 was used based on the "nearest neighbor" method [17]. The propensity scores were estimated using logistic regression which included the following variables: age, sex, body mass index (BMI), American Society of Anesthesiologists (ASA) score, level of serum alpha-fetoprotein (AFP), liver cirrhosis, and tumor characteristics (number and size). After 1 to 1 matching, 47 patients were included in the analyses in each group which had similar baseline and pathological characteristics. $P$ value $<0.05$ was considered as statistically significant.

\section{Results}

Baseline characteristics of the case (HS + LPMOD) and control (LPMOD only) group are presented in Table 1. The cases comprised 54 patients with a histopathologically confirmed malignant tumor, while 25 patients with malignant hepatic tumors in the control group. There was not a significant difference between two groups in terms of the distribution of sex, histopathologic malignancy, ASA score, AFP, liver cirrhosis, and common pathological characteristics. Significant differences were observed depending on the group; the case group was more likely to have a lower BMI $(P=0.02)$ and higher elderly population $(P=0.03)$ than the control group. After PSM analysis, however, two groups had similar clinicopathologic features.

In the overall cohort, the intraoperative blood loss was significantly diminished in the case group compared with the control group (mean, 158.5 vs. 221.5 $\mathrm{ml}, P<0.01)$. In addition, the HS + LPMOD group had a significantly shorter operative time in minutes than that in the LPMOD only group (mean, 202.4 vs. 265.7 min, $P<0.01$ ). In the PSM cohort, mean operative times were shorter in the HS + LPMOD group (mean, 210.5 vs. $265.7 \mathrm{~min}, P=0.02$ ), and the $\mathrm{HS}+$ LPMOD group experienced less intraoperative blood loss compared to the control group (mean, 169.4 vs. $221.5 \mathrm{ml}, P=0.03)$. There were no differences in blood transfusion rate, conversion to laparotomy, postoperative hospital stay, resection margin, and perioperative hospital mortality between the two groups either before or after PSM (Table 2).

The postoperative parameters of liver function increased dramatically postoperatively, but no significant differences were observed in the mean peak level of ALT, AST, TBIL, and PT between the two groups either 
Table 1 Demographic and pathologic factors before and after propensity score matching among the patients in the study

\begin{tabular}{|c|c|c|c|c|c|c|}
\hline & \multicolumn{3}{|l|}{ Entire cohort } & \multicolumn{3}{|c|}{ Propensity-matched cohort } \\
\hline & HS + LPMOD $(n=98)$ & LPMOD $(n=47)$ & $P$ value & HS + LPMOD $(n=47)$ & LPMOD $(n=47)$ & $P$ value \\
\hline Age, mean (SD) & $58.6(11.1)$ & $55.7(11.8)$ & $0.03^{*}$ & $56.4(10.7)$ & $55.7(11.8)$ & 0.36 \\
\hline Sex & & & 0.20 & & & 0.41 \\
\hline Male, n (\%) & $65(66.3)$ & $26(55.3)$ & & $22(46.8)$ & $26(55.3)$ & \\
\hline Female, n (\%) & $33(33.7)$ & $21(44.7)$ & & $25(53.2)$ & $21(44.7)$ & \\
\hline BMI, mean (SD) & $21.1(2.2)$ & $22.7(2.4)$ & $0.02^{*}$ & 21.8(1.9) & $22.7(2.4)$ & 0.34 \\
\hline ASA, n (\%) & & & 0.99 & & & 0.90 \\
\hline । & $28(28.6)$ & $13(27.7)$ & & $15(31.9)$ & $13(27.7)$ & \\
\hline$\|$ & $60(61.2)$ & $29(61.7)$ & & $27(57.4)$ & $29(61.7)$ & \\
\hline III & $10(10.2)$ & $5(10.6)$ & & $5(10.6)$ & $5(10.6)$ & \\
\hline AFP, n (\%) & & & 0.23 & & & 0.81 \\
\hline Increased ( $\geq 400$ ng/mL) & $15(15.3)$ & $11(23.4)$ & & $12(25.5)$ & $11(23.4)$ & \\
\hline Not increased $(<400 \mathrm{ng} / \mathrm{mL})$ & $83(84.7)$ & $36(76.6)$ & & $35(74.5)$ & $36(76.6)$ & \\
\hline Histopathologic diagnosis & & & 0.89 & & & 0.95 \\
\hline Colorectal carcinoma & $14(14.3)$ & $5(10.6)$ & & $5(10.6)$ & $5(10.6)$ & \\
\hline Hepatocellular Carcinoma & $36(36.7)$ & $19(40.4)$ & & $20(42.6)$ & $19(40.4)$ & \\
\hline Cholangiocarcinoma & $4(4.1)$ & $1(2.1)$ & & $2(4.3)$ & $1(2.1)$ & \\
\hline Benign & $38(38.8)$ & $20(42.6)$ & & $19(40.4)$ & $20(42.6)$ & \\
\hline Other & $6(6.1)$ & $2(4.3)$ & & $1(2.1)$ & $2(4.3)$ & \\
\hline Cirrhosis, n (\%) & $16(16.3)$ & $10(21.3)$ & 0.47 & $12(23.4)$ & $10(21.2)$ & 0.63 \\
\hline \multicolumn{7}{|l|}{ Pathologic characteristic } \\
\hline Number of tumors, n (SD) & $1.5(0.3)$ & $1.2(0.5)$ & 0.32 & $1.3(0.3)$ & $1.2(0.5)$ & 0.54 \\
\hline Largest tumor size, cm (SD) & $6.1(3.5)$ & $5.8(2.8)$ & 0.46 & $6.1(3.3)$ & $5.8(2.8)$ & 0.62 \\
\hline
\end{tabular}

Abbreviations: AFP alpha-fetoprotein, ASA American Society of Anesthesiologists, BMI body mass index, SD standard deviation. HS harmonic scalpel, $L P M O D$ laparoscopic Peng's multifunction operative dissector

${ }^{*} P<0.05$; ${ }^{*} P<0.01$ between two groups

before or after PSM (Table 3). The bile leakage rate was comparable between the two groups $(7.1 \%$ vs. $8.5 \%, P=$ 0.77 ; $6.4 \%$ vs. $8.5 \%, P=0.69$, respectively) before and after matching. The bile fistula was resolved following a short course of drainage and antibiotic therapy in both groups.

\section{Discussions}

Our study found that our two-handed technique combining HS and LPMOD in LHH can significantly reduce intraoperative blood loss and shorten operative time, which is of great clinical value. The proposed benefits of this novel laparoscopic techniques over traditional onehand hepatectomy techniques are numerous including quicker physical recovery and decreased postoperative pain and general preference by patients. Synergistic combinations of the advantages of different hepatectomy tools instead of individual hepatectomy technique per se not only optimize the surgical procedures but improve the safety and effectiveness of challenging hemihepatectomy through reducing intraoperative blood loss and shortening operative time which substantively increase the survival rate with better prognosis of patients with liver diseases, especially cancer patients.

In 2005, Aloia et al. [18] first reported a two-surgeon technique; two surgeons were allowed to employ two instruments to participate in the parenchymal transection for major hepatectomies. The primary surgeon dissected the liver parenchyma by ultrasonic dissection (UD) device from the left side of the patient. Simultaneously, the assistant surgeon held the saline-linked cautery (SLC) device that stood next to the patient's right side. Aloia showed that the "two-surgeon technique," using a combination of UD and SLC in hepatic resection resulted in shorter operative time and a reduction in the duration of hepatoduodenal ligament occlusion, while the postoperative liver function and complications rate were similar to that of the "one-surgeon technique" group. Mitsuhisa Takatsuki [19] claimed that the blood loss and donor complications in living donor hepatectomies significantly reduced when using the two-surgeon technique with Cavitron Ultrasonic Surgical Aspirator (CUSA) and SLC, while the early graft function and postoperative recipient survival did not differ between the two groups. This 
Table 2 Operative factors before and after propensity score matching among the patients in the study

\begin{tabular}{|c|c|c|c|c|c|c|}
\hline & \multicolumn{3}{|l|}{ Entire cohort } & \multicolumn{3}{|c|}{ Propensity-matched cohort } \\
\hline & HS + LPMOD $(n=98)$ & LPMOD $(n=47)$ & $P$ value & HS + LPMOD $(n=47)$ & LPMOD $(n=47)$ & $P$ value \\
\hline Hemihepatectomy & & & 0.58 & & & 0.68 \\
\hline Left (\%) & $59(60.2)$ & $21(44.7)$ & & $28(59.6)$ & $26(55.3)$ & \\
\hline Right (\%) & 39 (39.8) & $21(55.3)$ & & $19(40.4)$ & $21(44.7)$ & \\
\hline Blood loss, mL (SD) & $158.5(124.2)$ & $221.5(176.3)$ & $<0.01^{* *}$ & $169.4(133.5)$ & $221.5(176.3)$ & $0.03^{*}$ \\
\hline Blood transfusion, n (\%) & $26(26.5)$ & $13(27.7)$ & 0.72 & $14(29.8)$ & $13(27.7)$ & 0.82 \\
\hline Operative time, minutes (SD) & $202.4(47.5)$ & $265.7(67.1)$ & $<0.01^{* *}$ & $210.5(56.1)$ & $265.7(67.1)$ & $0.02^{*}$ \\
\hline Conversion to laparotomy, n (\%) & $5(5.1)$ & $3(6.4)$ & 0.75 & $4(8.5)$ & $3(6.4)$ & 0.69 \\
\hline Postoperative hospital stay, days (SD) & $14.1(9.4)$ & $13.7(9.4)$ & 0.80 & $14.0(8.7)$ & $13.7(9.4)$ & 0.83 \\
\hline Hospital mortality, n (\%) & 0 & 0 & - & 0 & 0 & - \\
\hline Resection margin\$ & & & 0.97 & & & 1.00 \\
\hline RO (\%) & $96(98.0)$ & $46(97.9)$ & & $46(97.9)$ & $46(97.9)$ & \\
\hline R1 (\%) & $2(2.0)$ & $1(2.1)$ & & $1(2.1)$ & $1(2.1)$ & \\
\hline R2 & 0 & 0 & & 0 & 0 & \\
\hline
\end{tabular}

Abbreviations: HS harmonic scalpel, LPMOD laparoscopic Peng's multifunction operative dissector, SD standard deviation

\$ Resection margin: R0: no microscopically identifiable tumor remnants, R1: microscopically identifiable tumor remnants R2: macroscopically identifiable tumor remnants

${ }^{*} P<0.05 ;{ }^{* *} P<0.01$ between two groups

finding might be explained by three reasons: (a) the "two-surgeon technique" provides surgeons with less time for exchanging surgical instruments for dissection and coagulation leading to the acceleration of the operation process and (b) the interactive participation of two surgeons during the parenchyma transection promoted the efficiency of surgical processes [20].

Accurate hemostasis is the key achievement in successful laparoscopic liver resection. It also depends on a tacit cooperation between the primary surgeon and assistants. Recent studies have investigated the twosurgeon technique to optimize the surgical procedure of $\mathrm{LHH}$ and indicated that it can notably ameliorate the efficiency of liver transection, shorten the operative time, and reduce intraoperative blood loss [18-20]. Nevertheless, cooperation between surgeons is essential to optimize the management of surgical procedures, which is always difficult. The learning curve is steep and the assistant surgeons demand a relatively long training period.
Inspired by the two-surgeon technique, our wellpracticed surgeon team started applying our two-handed technique, which combined LPMOD and HS to complete laparoscopic liver parenchyma transection. The operator could manipulate both the LPMOD and HS in real-time by himself to dissect the liver parenchyma in different parts of the transection plane. The two-handed technique can achieve the similar effect with the traditional hepatectomy method. Moreover, the accidental hemorrhage can be managed more accurately and immediately while the surgeon is well trained with both hands. The instructions for the two-handed operation issued by the primary surgeon were more precise and would not cause any misunderstanding which may occur among surgeons when performing the two-surgeon technique. The advantages of two-handed technique can be absolutely utilized by the surgeon during the operation without exchanging or passing instruments frequently. The improvement accelerates the surgical process

Table 3 Postoperative variables before and after propensity score matching

\begin{tabular}{|c|c|c|c|c|c|c|}
\hline & \multicolumn{3}{|l|}{ Entire cohort } & \multicolumn{3}{|c|}{ Propensity-matched cohort } \\
\hline & HS + LPMOD $(n=98)$ & LPMOD $(n=47)$ & $P$ value & HS + LPMOD $(n=47)$ & LPMOD $(n=47)$ & $P$ value \\
\hline Bile leakage n (\%) & $7(7.1)$ & $4(8.5)$ & 0.77 & $3(6.4)$ & $4(8.5)$ & 0.694 \\
\hline \multicolumn{7}{|l|}{ Liver functions } \\
\hline Mean peak AST ( \pm SD) & $253.8(146.4)$ & $316.7(187.8)$ & 0.33 & $260.8(156.5)$ & $316.7(187.8)$ & 0.431 \\
\hline Mean peak ALT ( \pm SD) & $237.2(126.1)$ & $280.7(223.6)$ & 0.21 & $242.0(143.2)$ & $280.7(223.6)$ & 0.232 \\
\hline Mean peak total bilirubin ( \pm SD) & $27.0(4.7)$ & $29.7(5.5)$ & 0.65 & $27.3(3.6)$ & $29.7(5.5)$ & 0.703 \\
\hline Mean peak prothrombin time $( \pm S D)$ & $15.3(3.4)$ & $15.1(2.5)$ & 0.24 & $15.2(3.1)$ & $15.1(2.5)$ & 0.262 \\
\hline
\end{tabular}

HS harmonic scalpel, $L P M O D$ laparoscopic Peng's multifunction operative dissector, ALT alanine transaminase, AST aspartate transaminase 
significantly and optimizes the quality of operation. However, this technique is accompanied by difficulties including adaptation of the non-dominant hand, so intensive trainings are usually required to master the technique. Meanwhile, the role of assistant surgeons cannot be neglected since they play critical roles in the field of vision exposure. The assisting surgeon can still use their free hand to employ an instrument to aid the surgery.

To our knowledge, this is the first study to evaluate the safety and effectiveness of the two-handed technique combined with HS and LPMOD for hepatic parenchyma transection in LHH. With similar baseline demographics and tumor characteristics, the two-handed technique significantly reduced blood loss and shortened operative time compared with the control group. Variables analyzed including blood transfusion, conversion to laparotomy, and the length of postoperative hospital stay and resection margin were comparable between the two groups. No significant differences were observed in postoperative liver function parameters between the two groups.

Hemorrhage during laparoscopic hemihepatectomy is mainly due to damage of the hepatic vein or branches inside the liver parenchyma [21]. Our two-handed technique can manage accidental hemorrhage perfectly since this technique combined the superiority of small vessels sealing function of HS with coordinating manipulation of LPMOD which is effective for hemostasis. It has been shown that massive blood loss is associated with an increased risk of death and recurrence after radical resection since serious intraoperative bleeding may facilitate dissemination of tumors, which could result in an increased risk of local recurrence [22-24].

Our study revealed that the two-handed technique group showed significantly shorter operative times than the classic technique group. This might be explained by the fact that in the "one hand" group, dissection of the vessels during liver parenchyma transection requires a supplementary procedure (harmonic scalpel dissection after clipping on the remnant side) to control bleeding at the sectioning plane, which cost extra time. However, with the help of the two-handed technique, HS can seal and occlude the small vessel simultaneously when ducts and vessels were exposed by LPMOD. Therefore, ultrasonic scissors that are combined with LPMOD could be shown to shorten the operation time and ameliorate the safety of vessel ligation.

There was no difference in the mean peak level of postoperative liver function measured by ALT, AST, TBIL, and PT between the two groups. The complication of bile leakage during hemihepatectomy may be affected by the tools chosen for parenchyma transection. In the two-handed technique group, bile leakage occurred in 3 patients (6.4\%) who were followed to be treated by long-time drainage without other invasive interventions. The incidence of bile leakage was comparable between the two groups $(6.4 \%$ vs. $8.5 \%)$ after PSM. Besides, there was no perioperative death in both groups. Therefore, our data suggested that the novel surgical techniques can also be safely and effectively performed in $\mathrm{LHH}$.

Our study is limited in several aspects. Firstly, this is a case-control study, so some potential selection bias might be concerned but this bias was minimized by applying PSM in the analyses and or controlled by that the study is nested in a prospectively collected database. Secondly, only patients who underwent LHH were included in this study so that our technique may not be generalized to other types of liver resection such as wedge resection and anatomical segmentectomy that were excluded in this study. Thirdly, surgeons are required to take some time to get trained and practiced if they want to use this technique. Future studies would need to assess the safety and effectiveness of the two-handed technique in other types of liver resection.

In conclusion, this study shows the new two-handed minimally invasive approach combining HS and LPMOD together provided patients with a safer and more effective technique of hepatic parenchymal transaction. Although no single technique is available to effectively complete the division and hemostasis during laparoscopic liver parenchyma transection, we can utilize the strengths of conceptually different hepatic transection instruments together to create a new operative technique like our two-handed technique. This technique can synergize the advantages of different hepatectomy tools, improve hepatic transection efficiency, optimize surgical procedures, reduce intraoperative blood loss, and shorten operative time. We strongly recommend this emerging technique to be applied in other laparoscopic hepatectomy institutions.

\section{Abbreviations \\ HS: Harmonic scalpel; LPMOD: Laparoscopic Peng's multifunction operative dissector; LHH: Laparoscopic hemihepatectomy; ALT: Alanine aminotransferase; AST: Aspartate transaminase; TBIL: Total bilirubin; PT: Prothrombin time; PSM: Propensity score matching; BMI: Body mass index; AFP: Alpha-fetoprotein}

\section{Acknowledgements}

The authors thank all colleagues of the department of general surgery in Sir Run Run Shaw Hospital of Zhejiang University.

\section{Authors' contributions}

$X L$ and $Y L L$ and contribute to the conception and design of the study; JWC and JHZ contribute to the analysis and interpretation of data. JWC, YYX, and GXJ contribute to drafting the article. The authors read and approved the final manuscript.

\section{Funding}

This research is supported by the National Natural Science Foundation of China (Grant No. 82072625).

Availability of data and materials

All the data can be obtained from the author by email (12018274@zju.edu.cn). 


\section{Declarations}

\section{Ethics approval and consent to participate}

This study was approved by the ethics committee of Sir Run Run Shaw Hospital. Written informed consent was obtained from all participants.

\section{Consent for publication}

Written informed consent was obtained from all patients enrolled in the investigation. The study protocol conformed to the ethical guidelines of the 1975 Declaration of Helsinki and the guidelines of the regional ethical committees of Sir Run Run Shaw Hospital, School of Medicine, Zhejiang University, China.

\section{Competing interests}

The authors declare that they have no competing interests.

Received: 2 February 2021 Accepted: 21 June 2021

Published online: 04 July 2021

\section{References}

1. Kawaguchi Y, Nomi T, Fuks D, Mal F, Kokudo N, Gayet B. Hemorrhage control for laparoscopic hepatectomy: technical details and predictive factors for intraoperative blood loss. Surgical Endoscopy. 2016;30(6):2543-51. https://doi.org/10.1007/s00464-015-4520-3.

2. Jubert AV, Lee ET, Hersh EM, McBride CM. Effects of surgery, anesthesia and intraoperative blood loss on immunocompetence. J Surg Res. 1973;15(6): 399-403. https://doi.org/10.1016/0022-4804(73)90110-8

3. Yamaguchi N, Yokoyama Y, Ebata T, Igami T, Sugawara G, Asahara T, et al. Intermittent Pringle maneuver is unlikely to induce bacterial translocation to the portal vein: a study using bacterium-specific ribosomal RNA-targeted reverse transcription-polymerase chain reaction. J Hepatobiliary Pancreat Sci. 2015;22(6):491-7. https://doi.org/10.1002/jhbp.239.

4. Katz SC, Shia J, Liau KH, Gonen M, Ruo L, Jarnagin WR, et al. Operative blood loss independently predicts recurrence and survival after resection of hepatocellular carcinoma. Ann Surg. 2009;249(4):617-23. https://doi.org/10.1 097/SLA.0b013e31819ed22f.

5. Zhang C, Wei HT, Hu W, Sun Y, Zhang Q, Abe M, et al. The feasibility of laparoscopic TSME preserving the left colic artery and superior rectal artery for upper rectal cancer. World J Surg Oncol. 2020;18(1):212. https://doi.org/1 0.1186/s12957-020-01986-6.

6. Grieco M, Apa D, Spoletini D, Grattarola E, Carlini M. Major vessel sealing in laparoscopic surgery for colorectal cancer: a single-center experience with 759 patients. World J Surg Oncol. 2018;16(1):101. https://doi.org/10.1186/s12 957-018-1402-x.

7. Cuk P, Simonsen RM, Komljen M, Nielsen MF, Helligsø P, Pedersen AK, et al. Improved perioperative outcomes and reduced inflammatory stress response in malignant robot-assisted colorectal resections: a retrospective cohort study of 298 patients. World J Surg Oncol. 2021;19(1):155. https://doi. org/10.1186/s12957-021-02263-W.

8. Huang ZS, Wu JW, Li Y, Lin YH, Li XY. Effect of sidedness on survival among patients with early-stage colon cancer: a SEER-based propensity score matching analysis. World J Surg Oncol. 2021;19(1):127. https://doi.org/10.11 86/s12957-021-02240-3.

9. Wang B, Wu Z, Zhang R, Chen Y, Dong J, Qi X. Retrospective analysis of safety and efficacy of enhanced recovery pathways in stage II-III colorectal cancer patients submitted to surgery and adjuvant therapy. World I Surg Oncol. 2021:19(1):99. https://doi.org/10.1186/s12957-021-02203-8.

10. Yoon Y, Han H, Cho J, Ahn K. Total laparoscopic liver resection for hepatocellular carcinoma located in all segments of the liver. Surg Endoscopy. 2010;24(7):1630-7. https://doi.org/10.1007/s00464-009-0823-6.

11. Cardinal J, Reddy S, Tsung A, Marsh J, Geller D. Laparoscopic major hepatectomy: pure laparoscopic approach versus hand-assisted technique. J Hepatobiliary Pancreat Sci. 2013;20(2):114-9. https://doi.org/10.1007/s00534012-0553-3.

12. Ikeda M, Hasegawa $K$, Sano K, Imamura H, Beck Y, Sugawara Y, et al. The vessel sealing system (LigaSure) in hepatic resection: a randomized controlled trial. Ann Surg. 2009;250(2):199-203. https://doi.org/10.1097/SLA. ob013e3181a334fo.

13. Lesurtel M, Selzner M, Petrowsky H, Mccormack L, Clavien P. How should transection of the liver be performed?: A prospective randomized study in 100 consecutive patients: comparing four different transection strategies.
Ann Surg. 2005;242(6):814-22. https://doi.org/10.1097/01.sla.0000189121.3 5617.d7.

14. Cai J, Zheng J, Xie Y, Kirih MA, Jiang G, Liang Y, et al. A novel simple intracorporeal Pringle maneuver for laparoscopic hemihepatectomy: how we do it. Surg Endosc. 2020.

15. Cai X, Duan L, Wang Y, Jiang W, Liang X, Yu H, et al. Laparoscopic hepatectomy by curettage and aspiration: a report of 855 cases. Surg Endosc. 2016;30(7):2904-13. https://doi.org/10.1007/s00464-015-4576-0.

16. Fong $Y$, Brennan $M$, Brown $K$, Heffernan $N$, Blumgart L. Drainage is unnecessary after elective liver resection. Am J Surg. 1996;171(1):158-62 https://doi.org/10.1016/S0002-9610(99)80092-0.

17. Guo S, Fraser MW. Propensity score analysis: statistical methods and applications: Sage; 2010

18. Aloia T, Zorzi D, Abdalla E, Vauthey J. Two-surgeon technique for hepatic parenchymal transection of the noncirrhotic liver using saline-linked cautery and ultrasonic dissection. Ann Surg. 2005;242(2):172-7. https://doi.org/10.1 097/01.sla.0000171300.62318.f4

19. Takatsuki M, Eguchi S, Yamanouchi K, Tokai H, Hidaka M, Soyama A, et al. Two-surgeon technique using saline-linked electric cautery and ultrasonic surgical aspirator in living donor hepatectomy: its safety and efficacy. Am J Surg. 2009;197(2):e25-7. https://doi.org/10.1016/j.amjsurg.2008.01.019.

20. Palavecino M, Kishi Y, Chun Y, Brown D, Gottumukkala V, Lichtiger B, et al. Two-surgeon technique of parenchymal transection contributes to reduced transfusion rate in patients undergoing major hepatectomy: analysis of 1,557 consecutive liver resections. Surgery. 2010;147(1):40-8. https://doi. org/10.1016/j.surg.2009.06.027.

21. Jayaraman S, Khakhar A, Yang H, Bainbridge D, Quan D. The association between central venous pressure, pneumoperitoneum, and venous carbon dioxide embolism in laparoscopic hepatectomy. Surgical Endoscopy. 2009; 23(10):2369-73. https://doi.org/10.1007/s00464-009-0359-9.

22. Fisher ER, Turnbull RB Jr. The cytologic demonstration and significance of tumor cells in the mesenteric venous blood in patients with colorectal carcinoma. Surg Gynecol Obstet. 1955:100(1):102-8.

23. Roberts $S$, Watne A, Mc GR, Mc GE, Cole WH. Technique and results of isolation of cancer cells from the circulating blood. AMA Arch Surg. 1958; 76(3):334-46. https://doi.org/10.1001/archsurg.1958.01280210004002.

24. Melendez JA, Arslan V, Fischer ME, Wuest $D$, Jarnagin WR, Fong Y, et al. Perioperative outcomes of major hepatic resections under low central venous pressure anesthesia: blood loss, blood transfusion, and the risk of postoperative renal dysfunction. J Am Coll Surgeons. 1998;187(6):620-5. https://doi.org/10.1016/S1072-7515(98)00240-3.

\section{Publisher's Note}

Springer Nature remains neutral with regard to jurisdictional claims in published maps and institutional affiliations.

Ready to submit your research? Choose BMC and benefit from:

- fast, convenient online submission

- thorough peer review by experienced researchers in your field

- rapid publication on acceptance

- support for research data, including large and complex data types

- gold Open Access which fosters wider collaboration and increased citations

- maximum visibility for your research: over $100 \mathrm{M}$ website views per year

At BMC, research is always in progress.

Learn more biomedcentral.com/submissions 